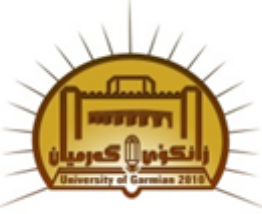

Available online at http://jgu.garmian.edu.krd

Journal of University of Garmian

https://doi.org/10.24271/garmian.196380

\title{
Solving Linear Fractional Programming Problem in symmetric trapezoidal fuzzy environment
}

\author{
Amir Sabir Majeed \\ Department of mechanic engineering, College of Technical Engineering, Sulaimani polytechnic University
}

\section{Article Info}

Received: June, 2019

Revised:August,2019

Accepted:September,2019

\section{Keywords}

fuzzy set, fuzzy number, trapezoidal fuzzy number, ranking function, Fuzzy mathematical programming, fuzzy linear programming, fuzzy fractional linear programming, Fuzzy simplex method.

\section{Corresponding Author}

amir.majeed@spu.edu.iq

\begin{abstract}
Fuzzy set theory has been applied on different important fields, such as managements, operations research, and control theory. In this paper, we provided a new algorithm to solving a fuzzy fractional linear programming problem (FFIPP) in full fuzzy environments. All the variables and coefficients of both the constraints and the objective function are symmetric trapezoidal fuzzy numbers. The fuzzy linear fractional programming problem (FLFP) has been switched to fuzzy linear programming (FLP) problems, where both of constraint and objective function involve fuzzy numbers as variables and coefficients. Further, using the fuzzy simplex method algorithm to get an optimal fuzzy solution. Finally, we provide illustrative numerical examples.
\end{abstract}




\section{Introduction}

The significance of fractional linear programming cames from the fact that many real-world problems are based on the ratio on financial, economic or physical values such as (cost/volume, profit /cost or cost/time) in production and financial planning [1]. Various application problems, can be derived as mathematical programming problems model, may be formulated with uncertainty. For several cases, the coefficients contained in either the objective or constraint functions are imprecise in nature and have to be considered as fuzzy numbers to reverse the real life situation the emanating mathematical problem is therefore indicated to as a fuzzy mathematical program-mix problem. The concept of using fuzzy numbers in mathematical programming was suggested in general for the first time by Tanaka et al. (1974a) [2] in the framing of the fuzzy decision of Bellman and Zadeh (1970) [3] when they presented building on fuzzy environment. There are several fractional programming model applications in fuzzy numbers as T.Peric, Z. Basic and S.Resic [4] ZIMMERMANN (1978) [5] proposed the first formulation of fuzzy linear programming and constructed a model of the problem also depend on the fuzzy concept of Bellman and Zadeh Fang and Hu [6] contemplated linear programming which its constraint coefficients are fuzzy numbers.

Vasant and et al [7] seek linear programming which its variables are fuzzy numbers for decision making in industrial production planning.

Ranking fuzzy number is also play important role in decision to making statistical analysis economic systems and operations research. So A.Nchammai and P.Thangaraj [8] solving intuitionist fuzzy linear programming by using metric distance ranking. Also H.M.Nehi and H.Hajmohamadi [9] provide a ranking function method used for solving fractional linear programming problems with multi - objective function. This paper is displayed as follows; section 1,provied the introduction in section 2, we provide the definition of both of fuzzy set and trapezoidal fuzzy numbers and some related properties . In section 3, we provide the arithmetic operation on symmetric fuzzy numbers and some other related definitions. In section 4, we give a definition of fuzzy fractional linear programming (FFLP) problem and the duality. In section 5, we explain the dual simplex method algorithm and finally we give some numerical example and our work conclusion.

\section{Preliminaries}

In this section, we have introduced some fundamental concepts of fuzzy sets and some kind of fuzzy numbers such as trapezoidal number, which was very useful in this paper.

\section{Fuzzy Sets}

Zadeh suggested a chain of membership functions that could be classified into two classes: those made up of straight lines being "linear" ones, and the "curved" represent "nonlinear" ones.

In addition, the nonlinear functions rise the time of computation. Therefore, in practice, most applications use linear fit functions. We will now continue to take some types of membership functions construct on Klir, at al [9],

Definition 2.1: A fuzzy set $\tilde{A}$ in $X$ (Universal Set) is defined as a set of an ordered pair $\tilde{A}=\left\{\left(x, \mu_{\tilde{A}}(x)\right)\right.$ $\mid x \in X \quad\}$, Where $\mu_{A}(x)$ defined as a membership function of the fuzzy set which maps $\mu_{\tilde{A}}(x): \rightarrow[0,1]$, for each $x \in X$

Definition 2.1.1: Support of a fuzzy set $\tilde{A}$ defind as a set of all $x \in \mathrm{X}$ with $\mu_{\tilde{A}}(x)>0$.

Definition 2.1.2: Core of a fuzzy set $\tilde{A}$ is a set of all $x \in X$ with $\mu_{\tilde{A}}(x)=1$.

Definition 2.1.3: A fuzzy set with non empty core set is called normal fuzzy set

Definition 2.2: An $\alpha$-cut of a fuzzy set $\tilde{A}$ is a crisp set that is defined as $\tilde{A} \alpha=\left\{x \in \mathbb{R} \mid \mu_{\tilde{A}}(x) \geq \alpha\right\}$ and it is also called (level subset $-\alpha$ ).

Definition 2.3: A strong $\alpha$ - cut (strong level subset- $\alpha$ ) is defined as $\tilde{A} \alpha>=\left\{x \in X \mid \mu_{\tilde{A}}(x)>\alpha\right\}$.

Definition 2.4: A fuzzy set $\tilde{A}$ called convex fuzzy set if for any $x \in X$ and $\delta \in[0,1]$ then:

$\mu_{\tilde{A}}(\delta x+(1-\delta)) \geq \min \left\{\mu_{\tilde{A}}(x), \mu_{\tilde{A}}(y)\right\}$.

Remarks:

1) A fuzzy set $\widetilde{A}$ is called convex set if and only if all of the non-empty $\alpha$-cut are convex.

2) A fuzzy number $\tilde{A}$ is a normal and convex fuzzy set.

3) There are some kinds of fuzzy numbers for instance a trapezoidal fuzzy number which it defined as follow:

Definition 2.5: Trapezoidal function defined by its minimum limit and its maximum limit $\mathrm{d}$, and the lower and upper values of its nucleus, $\mathrm{b}$ and $\mathrm{c}$ respectively.

$\mu_{a}=\left\{\begin{array}{cl}0 & \text { if }(x \leq a) \text { or }(x \geq d) \\ \frac{x-a}{b-a} & \text { if } x \in(a, b] \\ 1 & \text { if } x \in(a, b) \\ \frac{d-x}{d-c} & \text { if } x \in[c, d)\end{array}\right.$

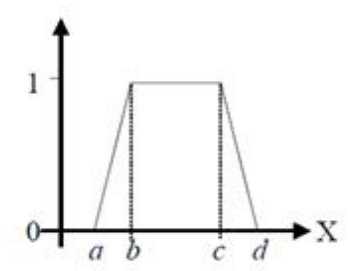


The fuzzy number with the above membership is called trapezoidal fuzzy number and it denoted by $\tilde{A}=(b, c, a, d)$

Remarks:

1) If $b=c$ then the fuzzy is called triangle fuzzy number

2) If $(b-a)=(d-c)$ the trapezoidal number is known as a symmetric

I.e. we can define a symmetric trapezoidal fuzzy number as a fuzzy number on (real numbers) $\mathbb{R}$

If there exist $a_{1}, a_{2}, \alpha \in \mathbb{R}$, when $a_{1} \leq a_{2}$ and $\alpha>$ 0 such that

$\mu_{\tilde{a}}=\left\{\begin{array}{cc}\frac{x}{\alpha}+\frac{\alpha-a_{1}}{\alpha} & \text { if } x \in\left(a_{1}-\alpha, a_{1}\right] \\ 1 & \text { if } x \in\left(a_{1}, a_{2}\right) \\ \frac{-x}{\alpha}+\frac{a_{2}+\alpha}{\alpha} & \text { if } x \in\left[a_{2}, a_{2}+\alpha\right) \\ 0 & \text { otherwise }\end{array}\right.$

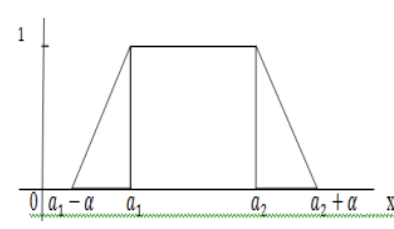

Then we write the symmetric fuzzy number as $\tilde{a}=\left(a_{1}, a_{2}, \alpha, \alpha\right)$, with $\left[a_{1}, a_{2}\right]$ as its core and $\left[a_{1}-\alpha, a_{2}+\alpha\right]$ is the support of $\tilde{a}$ and $\mathcal{F}(\mathbb{R})$ is a set of all symmetric trapezoidal fuzzy numbers, where $\mu_{\tilde{a}}$ is a membership of $\tilde{a}$

\section{Arithmetical Operation on Symmetric Fuzzy} Numbers:

Definition 3.1: Let $\quad \tilde{a}, \tilde{b} \in \mathcal{F}(\mathbb{R})$ and $\quad \tilde{a}=$ $\left(a_{1}, a_{2}, \alpha, \alpha\right), \tilde{b}=\left(b_{1}, b_{2}, \beta, \beta\right)$ then

1. The relation $\tilde{a} \leqslant \tilde{b}$ if and only if $\frac{\left(a_{1}-\alpha\right)+\left(a_{2}+\alpha\right)}{2}<\frac{\left(b_{1}-\beta\right)+\left(b_{2}+\beta\right)}{2}$

Then we face the following cases:

i) $\quad \frac{a_{1}+a_{2}}{2}<\frac{b_{1}+b_{2}}{2}$ (in this case we can write $\tilde{a} \prec$ $\tilde{b})$

ii) $O r \quad \frac{a_{1}+a_{2}}{2}=\frac{b_{1}+b_{2}}{2}, b_{1}<a_{1}$ and $a_{2}<b_{2}$

iii) $O r \quad \frac{a_{1}+a_{2}}{2}=\frac{b_{1}+b_{2}}{2}, b_{1}=a_{1}, a_{2}=b_{2}$ and $\alpha<$ $\beta$

(In the cases (ii) and (iii), we can write $\tilde{a} \approx \tilde{b}$ which means that $\tilde{a}$ and $\tilde{b}$ are equivalent)

Remark 1: if $\tilde{a} \approx \tilde{b}$ where $\tilde{a}=\left(a_{1}, a_{2}, \alpha, \alpha\right)$ and $\tilde{b}=\left(b_{1}, b_{2}, \beta, \beta\right)$ is not need to be $a_{1}=b_{1}$ or $a_{2}=$ $b_{2}$

but $\left(a_{1}, a_{2}, \alpha, \alpha\right)-\left(b_{1}, b_{2}, \beta, \beta\right) \approx(-h, h, \alpha+\beta, \alpha+$ $\beta)$, where $h=\left(b_{2}-a_{1}\right) \geq 0$
Remark 2: For $\tilde{0} \in \mathcal{F}(\mathbb{R})$ we define $\tilde{0}$ by $(-c, c, \delta, \delta)$ if there exists $c, \delta>0$

Remark 3: For $\tilde{1} \in \mathcal{F}(\mathbb{R})$ we define $\tilde{1}$ by $(-c+$ $1, c+1, \delta, \delta)$ if there exists $c, \delta>0$, also $\tilde{1} \approx$ $(1,1,0,0)$

Definition 3.2: Let $\tilde{a}, \tilde{b}, \tilde{c} \in \mathcal{F}(\mathbb{R})$ then we can define the followings:

i) If $\tilde{a} \preccurlyeq \tilde{b} \operatorname{then} \begin{cases}\tilde{c} \tilde{a} \preccurlyeq \tilde{c} \tilde{b} & \text { if } \tilde{c} \geqslant \tilde{0} \\ \tilde{c} \tilde{a} \geqslant \tilde{c} \tilde{b} & \text { if } \tilde{c} \leqslant \tilde{0}\end{cases}$

ii) $\quad \tilde{c}(\tilde{a}+\tilde{b}) \approx \tilde{c} \tilde{a}+\tilde{c} \tilde{b}$ and $\tilde{c}(\tilde{a}-$ $\tilde{b}) \approx \tilde{c} \tilde{a}-\tilde{c} \tilde{b}$

iii) $\quad \tilde{a} \tilde{b} \geqslant \tilde{0}$ if and only if both of $\tilde{a} \geqslant \tilde{0}$ and $\tilde{b} \geqslant \tilde{0}$

Or both of $\tilde{a} \preccurlyeq \tilde{0}$ and $\tilde{b} \preccurlyeq \tilde{0}$

iv) $\quad \tilde{a} \tilde{b} \leqslant \tilde{0}$ if and only if $\tilde{a} \geqslant \tilde{0}$ and $\widetilde{b \preccurlyeq 0}$

Or $\tilde{a} \preccurlyeq \tilde{0}$ and $\widetilde{b} \geqslant \tilde{0}$

1. if $\mathrm{x} \in \mathbb{R}^{+}$then $x \tilde{a}=\left(x a_{1}, x a_{2}, x \alpha, x \alpha\right)$

2. if $\mathrm{x} \in \mathbb{R}^{-}$then $x \tilde{a}=\left(x a_{2}, x a_{1},-x \alpha,-x \alpha\right)$

3. $\tilde{a}+\tilde{b}=\left(a_{1}, a_{2}, \alpha, \alpha\right)+\left(b_{1}, b_{2}, \beta, \beta\right)=\left(a_{1}+b_{1}, a_{2}+\right.$ $\left.b_{2}, \alpha+\beta, \alpha+\beta\right)$

$\tilde{a}-\tilde{b}=\left(a_{1}, a_{2}, \alpha, \alpha\right)-\left(b_{1}, b_{2}, \beta, \beta\right)=\left(a_{1}-b_{2}, a_{2}-\right.$ $\left.b_{1}, \alpha+\beta, \alpha+\beta\right)$

4. $\tilde{a} * \tilde{b}=\left(a_{1}, a_{2}, \alpha, \alpha\right) *\left(b_{1}, b_{2}, \beta, \beta\right)$

$=\left(\left(\frac{a_{1}+a_{2}}{2}\right)\left(\frac{b_{1}+b_{2}}{2}\right)-\omega,\left(\frac{a_{1}+a_{2}}{2}\right)\left(\frac{b_{1}+b_{2}}{2}\right)+\right.$

$\left.\omega,\left|a_{2} \beta+b_{2} \alpha\right|,\left|\omega, a_{2} \beta+b_{2} \alpha\right|\right)$

Where

$\omega=\frac{h-k}{2}, h=\max \left(a_{1} b_{1}, a_{1} b_{2}, a_{2} b_{1}, a_{2} b_{2}\right) \quad$ and $k=\min \left(a_{1} b_{1}, a_{1} b_{2}, a_{2} b_{1}, a_{2} b_{2}\right)$

5. Inverse: $\quad \frac{1}{\tilde{a}}=\tilde{a}^{-1} \approx\left(\frac{2}{a_{1}+a_{2}}-\omega, \frac{2}{a_{1}+a}+\right.$ $\omega, \alpha, \alpha)$ where

Where $\omega=\frac{h-k}{2}, h=\max _{\mathrm{i}, \mathrm{j}=1,2}\left(\frac{1}{a_{j}}\right) \quad$ and $k=\min _{\mathrm{i}, \mathrm{j}=1,2}\left(\frac{1}{a_{j}}\right)$

And $\frac{1}{a_{j}}=\left\{\begin{aligned} \frac{1}{a_{j}} & \text { if } a_{j}>0 \\ 0 & \text { if } a_{j}=0\end{aligned}\right.$

6. Division: $\quad \frac{\tilde{a}}{\tilde{b}} \approx\left(\frac{a_{1}+a_{2}}{b_{1}+b_{2}}-\omega, \frac{a_{1}+a_{2}}{b_{1}+b_{2}}+\omega, \mid a_{2} \beta+\right.$ $\left.\frac{\alpha}{b_{2}}|,| a_{2} \beta+\frac{\alpha}{b_{2}} \mid\right)$

Where $\quad \omega=\frac{h-k}{2}, h=\max _{\mathrm{i}, \mathrm{j}=1,2}\left(\frac{a_{i}}{b_{j}}\right) \quad$ and $\quad k=$ $\min _{\mathrm{i}, \mathrm{j}=1,2}\left(\frac{a_{i}}{b_{j}}\right)$

And: $\frac{\tilde{a}}{\tilde{a}} \approx\left(\frac{a_{1}+a_{2}}{a_{1}+a_{2}}-\omega, \frac{a_{1}+a_{2}}{a_{1}+a_{2}}+\omega,\left|a_{2} \beta+\frac{\alpha}{a_{2}}\right|,\left|a_{2} \beta+\frac{\alpha}{a_{2}}\right|\right)$ $\approx\left(1-\omega, 1+\omega,\left|a_{2} \beta+\frac{\alpha}{a_{2}}\right|,\left|a_{2} \beta+\frac{\alpha}{a_{2}}\right|\right) \approx \tilde{1}$ 


\section{3: Definitions:}

We introduce the notation of a fuzzy matrix:

Definition3.3.1: A matrix $A_{F}=\left(a_{i j}\right)_{m \times n}$, where $a_{i j} \forall i, j$ is a fuzzy number is called a fuzz matrix

Definition3.3.2: A matrix $A_{F}=\left(a_{i j}\right)_{m \times 1}$, where $a_{i j} \forall i, j$ is a fuzzy number is called a fuzz vector (one column matrix)

Definition3.3.3: any matrix of A symmetric trapezoidal fuzzy numbers $\widetilde{A_{F}}=\left(\tilde{a}_{i j}\right)_{m \times n}$ where $a_{i j} \in \mathcal{F}(\mathbb{R}), i=$ $1,2, \ldots m, j=1,2 \ldots n$ is called $A$ symmetric trapezoidal fuzzy matrix and the set of all A symmetric trapezoidal fuzzy matrices is denoted by $\mathcal{F}(\mathbb{R})^{m \times n}$

Definition3.3.4: corresponding of any square symmetric trapezoidal fuzzy matrix

$\widetilde{A_{F}} \in \mathcal{F}(\mathbb{R})^{n \times n}$, there is A symmetric trapezoidal fuzzy number which is called determinant of $\widetilde{A_{F}}$ and is denoted by $\left|\widetilde{A_{F}}\right|$ which computed as follows:

$$
\left|\widetilde{A_{F}}\right|=
$$$$
\left\{\begin{array}{l}
\tilde{a}_{11} \\
\tilde{a}_{11} \tilde{a}_{22}-\tilde{a}_{12} \tilde{a}_{21} \\
\sum_{j=1}^{n}(-1)^{i+j} \tilde{a}_{i j}\left|\widetilde{A_{l \jmath}}\right|, \text { for any } i=1,2 \ldots n
\end{array}\right.
$$

for $n=1$ for $n=2$

for $n>2$

Remark: $A$ matrix $\widetilde{A_{F}}=\left(\tilde{a}_{i j}\right)_{n \times n}$ is called singular if $\left|\widetilde{A_{F}}\right|=\tilde{0}$, otherwise is called non-singular

Definition3.3.5:

let

$\widetilde{A_{F}} \in \mathcal{F}(\mathbb{R})^{n \times n}$ be a square matrix then

the matrix $\widetilde{B_{F}}$

$\in \mathcal{F}(\mathbb{R})^{n \times n}$ is called invers of $\widetilde{A_{F}}$, denoted by $\widetilde{B_{F}}$

$={\widetilde{A_{F}}}^{-1}$

if $\widetilde{B_{F}} * \widetilde{A_{F}} \approx \widetilde{A_{F}} * \widetilde{B_{F}} \approx \widetilde{I_{n}}$, where $\widetilde{I_{n}}$ is an identity matrix.

Also ${\widetilde{A_{F}}}^{-1}$ is calculated

by ${\widetilde{A_{F}}}^{-1}=\frac{(1,1,0,0)}{\left|\widetilde{A_{F}}\right|}\left[(-1)^{i+j}\left|\widetilde{A_{l j}}\right|\right]_{n \times n}$,

Where $\widetilde{A_{\imath j}}$ be the (ij)th minor of the matrix

Definition3.3.6: the ranking function is defined as a function which maps for each fuzzy number in to the real line denoted by $\mathfrak{R}: \mathcal{F}(\mathbb{R}) \quad \mathbb{R}$

By Maleki ranking function suggestion [10]

Let $\tilde{A}=\left(a_{1}, a_{2}, \alpha, \beta\right)$ be a trapezoidal fuzzy number then

$\mathfrak{R}(\tilde{A})=a_{1}+a_{2}+\frac{1}{2}(\beta-\alpha)$

Remark 1: $\mathfrak{R}(\tilde{A})=a_{1}+a_{2}$ where $\tilde{A}$ be a symmetric trapezoidal fuzzy number

Remark2: let $\quad \tilde{A}=\left(a_{1}, a_{2}, a_{3}, a_{4}\right)$ and $\tilde{B}=$ $\left(b_{1}, b_{2}, b_{3}, b_{4}\right)$ be two trapezoidal fuzzy numbers then:

i) $\quad \tilde{A} \underset{\mathfrak{R}}{\geq} \tilde{B}$ if and only if $\mathfrak{R}(\tilde{A}) \geq \mathfrak{R}(\tilde{B})$

(1) ii) $\quad \tilde{A} \overline{\bar{R}} \tilde{B}$ if and only if $\mathfrak{R}(\tilde{A})=$ $\Re(\tilde{B})$

iii) $\quad \tilde{A} \leq \tilde{B}$ if and only if $\Re(\tilde{A}) \leq$ $\Re(\widetilde{B})$

\section{Fully fuzzy fractional linear programming problems:}

A fully fuzzy fractional linear programming (FFFLP) problem with symmetric trapezoidal fuzzy numbers can be defined as:

$$
\begin{gathered}
\text { Max } \tilde{Z}=\frac{\tilde{c} \tilde{x}+\tilde{p}}{\tilde{d} \tilde{x}+\tilde{q}} \\
\text { Subject to } \\
\widetilde{A_{F}} \tilde{x} \preccurlyeq \widetilde{B_{F}} \\
\tilde{x} \geqslant \tilde{0}
\end{gathered}
$$

Where $\widetilde{B_{F}} \in \mathcal{F}(\mathbb{R})^{m}, \tilde{x} \in \mathcal{F}(\mathbb{R})^{n}, \tilde{A} \in \mathcal{F}(\mathbb{R})^{m \times n}$ and $\tilde{c}^{T}, \tilde{d}^{T}, \tilde{p}^{T}, \tilde{q}^{T} \in \mathcal{F}(\mathbb{R})^{n}$

Definition4.1 A fully fuzzy linear programming (FFLP) problems with symmetric trapezoidal fuzzy

numbers is defined as:

$\operatorname{Max} \tilde{Z}=\tilde{c} \tilde{x}$
Subject to

$$
\begin{aligned}
& \widetilde{A_{F}} \tilde{x} \preccurlyeq \widetilde{B_{F}} \\
& \tilde{x} \geqslant \tilde{0}
\end{aligned}
$$

Where $\widetilde{B_{F}} \in \mathcal{F}(\mathbb{R})^{m}, \tilde{x} \in \mathcal{F}(\mathbb{R})^{n}, \widetilde{A_{F}} \in \mathcal{F}(\mathbb{R})^{m \times n}$ and $\tilde{c}^{T} \in \mathcal{F}(\mathbb{R})^{n}$

Definition4.3: a feasible solution to $(F F F L P)$ problem is a fuzzy vector

$\tilde{x}=\left(\tilde{x}_{1}, \tilde{x}_{2}, \tilde{x}_{3}, \ldots \tilde{x}_{n}\right)^{T} \in \mathcal{F}(\mathbb{R})^{n}$, where each $\tilde{x}_{n} \in$ $\mathcal{F}(\mathbb{R})$, if $\tilde{x} \geqslant \tilde{0}$

satisfy the constraints $\tilde{A} \tilde{x} \leqslant \tilde{b}$.

Definition4.1 A fully fuzzy linear programming (FFLP) problems with symmetric trapezoidal fuzzy numbers is defined as:

Max

$$
\tilde{Z}=\tilde{c} \tilde{x}
$$

Subject to

$$
\begin{aligned}
\widetilde{A_{F}} \tilde{x} & \preccurlyeq \widetilde{B_{F}} \\
\tilde{x} & \geqslant \tilde{0}
\end{aligned}
$$

Where $\widetilde{B_{F}} \in \mathcal{F}(\mathbb{R})^{m}, \tilde{x} \in \mathcal{F}(\mathbb{R})^{n}, \widetilde{A_{F}} \in \mathcal{F}(\mathbb{R})^{m \times n}$ and $\tilde{c}^{T} \in \mathcal{F}(\mathbb{R})^{n}$

Definition4.2: we define the dual problem of (FFLP) as follows:

Min

$$
\tilde{u} \approx \widetilde{w} \tilde{b}
$$

(6)

Subject to

$$
\begin{aligned}
& \tilde{w} \tilde{A} \geqslant \tilde{c} \\
& \widetilde{W} \geqslant \tilde{0}
\end{aligned}
$$

And denoted by (DFFLP) 
Duality properties:

i) weak duality properties: if $\tilde{x}$ and $\widetilde{w}$ are feasible solution to fully fuzzy linear programming (FFLP) and dual fully fuzzylinear programming(DFFLP) respectively, then $\widetilde{w} \tilde{b} \geqslant \tilde{c} \tilde{x}$

ii) strong duality properties: if any one of (FFLP) and (DFFLP) has a fuzzy optimal solution then both of them have fuzzy optimal solutions and the fuzzy optimal objective values are equal

iii)(complementary slackness): if $\tilde{x}_{*}$ be any fuzzy feasible solution to (FFLP) and $\widetilde{w}_{*}$ be any fuzzy feasible solution to (DFFLP) problems .then

$\left(\widetilde{W}_{*} \tilde{A}-\tilde{c}\right) \tilde{x}_{*} \approx \tilde{0}, \widetilde{W}_{*}\left(\tilde{b}-\tilde{A} \tilde{x}_{*}\right) \approx \tilde{0}$ If and only if $\tilde{x}_{*}$ and $\widetilde{w}_{*}$ are fuzzy optimal solution of them respectively.

iv) (Unboundnity properties): the (FFLP) OR (DFFLP) problem is unbounded if fuzzy feasible solutions exist such that the fuzzy objective value is increased or decreased indefinitely.

Remark: if any one of the (FFLP) or (DFFLP) problems is unbounded, then the other problem has no fuzzy feasible solution.

Definition4.3: a feasible solution to (FFFLP) problem is a fuzzy vector

$\tilde{x}=\left(\tilde{x}_{1}, \tilde{x}_{2}, \tilde{x}_{3}, \ldots \tilde{x}_{n}\right)^{T} \in \mathcal{F}(\mathbb{R})^{n}$, where each $\tilde{x}_{n} \in$

$\mathcal{F}(\mathbb{R})$, if $\tilde{x} \geqslant \tilde{0}$

satisfy the constraints $\tilde{A} \tilde{x} \preccurlyeq \tilde{b}$.

Definition4.4: a fuzzy optimal solution to (FFFLP) is a

fuzzy feasible solution

$\tilde{x}_{*} \in$

$S$ where $S$ be the set of all feasible solution to

(FFFLP)

and $\tilde{c} \tilde{x} \preccurlyeq \tilde{c} \tilde{x}_{*} \forall \tilde{x} \in S$

Fuzzy basic feasible solution: we introduce the fuzzy basic feasible solution of the fuzzy (LP) with fuzzy numbers and variables as follows:-

Consider the standard (FFLP) as

$\operatorname{Max} \tilde{Z}=\tilde{c} \tilde{x}$

Subject to $\tilde{A} \tilde{x} \overline{\bar{R}} \tilde{b}$ and $\tilde{x}_{B} \geq \tilde{0}$

Where $\tilde{A}=\left[\tilde{a}_{i j}\right]_{m \times n^{\prime}}$,

Assume $\operatorname{rank}(\tilde{A})$

$=m$. partition $\widetilde{A}$, rerranging columns of $\tilde{A}$, if needed,

as $\left[\begin{array}{ll}\tilde{B} & \widetilde{N}\end{array}\right]$, where $\tilde{B}_{m \times m}$

and $|\tilde{B}| ¥ 0$ 0.It is obvious that $\operatorname{rank}(\tilde{B})=m$,

let $\tilde{y}_{j}$ be the solution to $\tilde{B} \tilde{y}$

$=\tilde{a}_{J}$, where $\tilde{a}_{J}$ is the jth column of the

coefficient matrix $\tilde{A}$.

it is clearlly that the basic solution

$\tilde{x}_{B} \approx\left(\tilde{x}_{B 1}, \tilde{x}_{B 2}, \tilde{x}_{B 3}, \ldots, \tilde{x}_{B m}\right)^{T} \overline{\bar{\Re}} \tilde{B}^{-1} \tilde{b} \overline{\bar{\Re}} \tilde{y}_{o}, \tilde{x}_{N} \overline{\bar{\Re}} \tilde{0}$

(8)

is a solution of $\tilde{A} \tilde{x} \quad \overline{\bar{\Re}} \quad \tilde{b}$. We call $\tilde{x}$ according partitioned as

$\left(\tilde{x}_{B}{ }^{T}, \tilde{x}_{N}{ }^{T}\right)^{T}$, a fuzzy basic solution corresponding to the basis $\tilde{B}$. if $\tilde{x}_{B} \underset{\Re}{\grave{0}}$, then the fuzzy basic solution is feasible and corresponding fuzzy objective value is $\tilde{z} \quad \overline{\bar{\Re}} \widetilde{C_{B}} \widetilde{x_{B}}$ where $C_{B}=\left(C_{B 1}, C_{B 2}, \ldots, C_{B n}\right)$

Now, corresponding to every fuzzy non-basic variable $\tilde{x}_{j}, 1 \leq j \leq n, j \neq \tilde{B}_{I}, I=1, \ldots, m$.

Define : $\tilde{z}_{j} \approx \tilde{c}_{B} \tilde{y}_{j} \approx \tilde{c}_{B} \tilde{B}^{-1}$

Remark: if $\tilde{x}_{B} \underset{\mathfrak{R}}{\geq} \tilde{0}$ then $\tilde{x}$ called nondegenerate fuzzy basic feasible solution, and if at least one component of $\tilde{x}_{B}$ is zero, then $\tilde{x}$ is called generate fuzzy basic feasible solution

\section{Fuzzy Dual Simplex Method}

\subsection{Primal Optimality and Dual feasibility}

Consider the following FFLP problem

$\operatorname{Max} \tilde{z}=\tilde{c} \tilde{x}$

(10)

Subject to

$$
\begin{aligned}
& \tilde{A} \tilde{x} \geqslant \tilde{b} \\
& \tilde{x} \geqslant \tilde{0}
\end{aligned}
$$

By introducing the nonnegative slack variables $\tilde{s}_{i, i}=1, \ldots, m$ for the ith constraint, we can rewrite (10) as follows:

$\operatorname{Max} \tilde{z}=\tilde{c} \tilde{x}+\tilde{0} \tilde{s}_{i}$,

Subject to

$$
\begin{array}{r}
\tilde{A} \tilde{x}-\tilde{0} \tilde{s}_{i,} \approx \tilde{b} \\
\tilde{x}, \tilde{s}_{i,} \geqslant \tilde{0}
\end{array}
$$

Where $\tilde{s} \approx\left(\tilde{s}_{1}, \tilde{s}_{2}, \ldots \tilde{s}_{m}\right)^{T}$,

Define $\overline{\tilde{x}}, \overline{\tilde{x}}^{T} \in(F(\mathbb{R}))^{n+m}$ as

$\overline{\tilde{x}}_{J}=\left\{\begin{array}{lr}\tilde{x}_{j}, & j=1,2, \ldots m \\ \tilde{s}_{j-n}, & j=n+1, \ldots n+m\end{array}\right\}$,

$\overline{\tilde{c}_{j}}=\left\{\begin{array}{lr}\tilde{c}_{j}, & j=1,2, \ldots n \\ \tilde{0}, & j=n+1, \ldots n+m\end{array}\right\}$

Assume that a basic solution for (11) is given by $\overline{\tilde{x}_{B}}=\tilde{B}^{-1} \tilde{b}$, with the basis matrix $\tilde{B}$.

Algorithm using dual simplex method:

Consider (FFFLP) problems with symmetric trapezoidal fuzzy numbers

$$
\operatorname{Max} \tilde{Z}=\frac{\tilde{c} \tilde{x}+\tilde{p}}{\tilde{d} \tilde{x}+\tilde{q}}
$$

Subject to

$$
\begin{gathered}
\widetilde{A_{F}} \tilde{x} \preccurlyeq \widetilde{B_{F}} \\
\tilde{x} \geqslant \tilde{0}
\end{gathered}
$$

Where $\quad \widetilde{B_{F}} \in \quad \mathcal{F}(\mathbb{R})^{m}, \tilde{x} \in \mathcal{F}(\mathbb{R})^{n}, \tilde{A} \in$ $\mathcal{F}(\mathbb{R})^{m \times n}$ and $\tilde{c}^{T}, \tilde{d}^{T}, \tilde{p}^{T}, \tilde{q}^{T} \in \mathcal{F}(\mathbb{R})^{n}$ 
Step1: analyze the objective function to two separate linear functions, the first function is represent the numerator function as

$\max \tilde{z}=\tilde{c} \tilde{x}+\tilde{p}$

Subject to

$$
\begin{gathered}
\widetilde{A_{F}} \tilde{x} \preccurlyeq \widetilde{B_{F}} \\
\tilde{x} \geqslant \tilde{0}
\end{gathered}
$$

And the second function is represent the denominator function as

$\min \tilde{z}=\tilde{d} \tilde{x}+\tilde{q}$

\section{Subject to}

$$
\begin{gathered}
\widetilde{A_{F}} \tilde{x} \leqslant \widetilde{B_{F}} \\
\tilde{x} \geqslant \tilde{0}
\end{gathered}
$$

Step2: reform a function max $\left(\tilde{z}^{*}(x)\right)$ from subtracting the denominator from the nominator and we put the resultant function in mathematical module made up of original restriction of problem with non-negativity condition and we rewrite it as:

$(F F L P) \operatorname{Max} \tilde{z}^{*}=\tilde{e} \tilde{z}+\tilde{h}$

Subject to

$$
\begin{gathered}
\widetilde{A_{F}} \tilde{x} \preccurlyeq \widetilde{B_{F}} \\
\tilde{x} \geqslant 0
\end{gathered}
$$

Where $\quad \widetilde{B_{F}} \in \quad \mathcal{F}(\mathbb{R})^{m}, \quad \tilde{x} \in \mathcal{F}(\mathbb{R})^{n}, \tilde{A} \in$ $\mathcal{F}(\mathbb{R})^{m \times n}$ and $\tilde{e}^{T}, \tilde{h}^{T} \in \mathcal{F}(\mathbb{R})^{n}$

Step3 :( Dual feasibility) Let $\widetilde{B_{F}}$ be a basis for the (FFLP) problem such that

$\tilde{x_{j}}-\tilde{c}_{j}$

$\geqslant \tilde{0}$ for all $j$, compute the simplex tableau.

If $\tilde{y}_{0} \geqslant \tilde{0}$ then the current solution is optimal otherwise select the pivot row $r$ with $\tilde{y}_{0 r} \prec \tilde{0}$

Step4: If $\tilde{y}_{r j} \geqslant \tilde{0}$ for all jthen the primal (FFLP) is infeasible otherwise select the pivot column $k$ by means of the following maximum ratio test:

$\frac{\tilde{z}_{k}-\tilde{c}_{k}}{\tilde{y}_{r k}} \approx \max \left\{\frac{\tilde{z}_{k}-\tilde{c}_{k}}{\tilde{y}_{r k}}: \tilde{y}_{r j} \prec \tilde{0}\right\}$ Pivot on $\tilde{y}_{r k}$

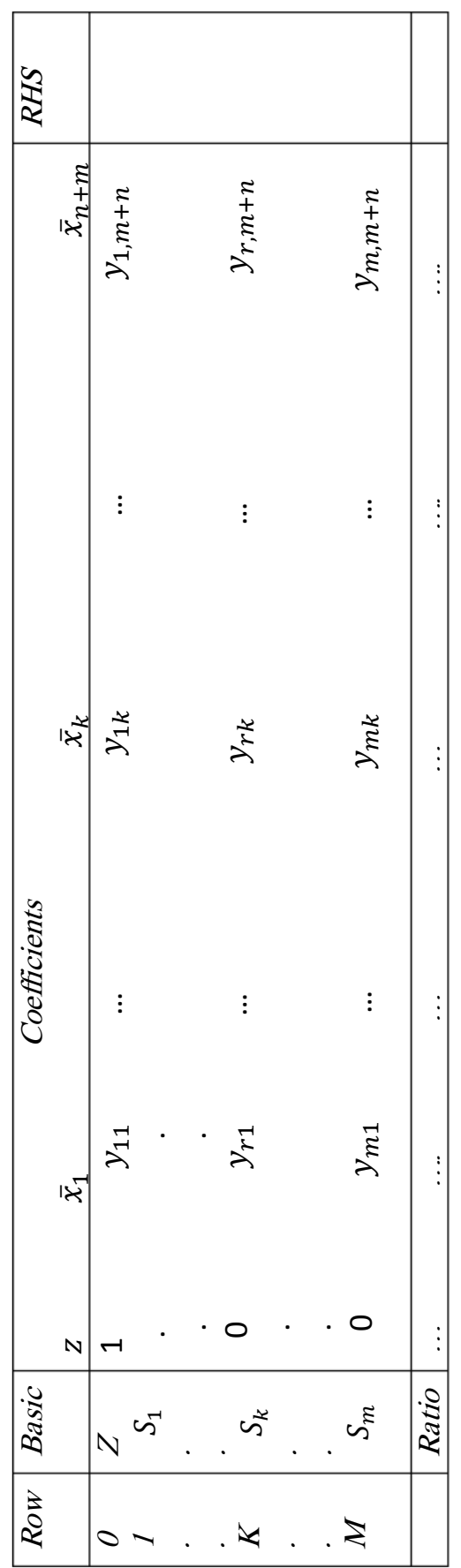

Numerical example1:

$\operatorname{Max} \tilde{Z}$

$=\frac{\left(4,6, \frac{1}{3}, \frac{1}{3}\right) \tilde{x}_{1}+(5,6,1,1) \tilde{x}_{2}+\left(5,6, \frac{1}{2}, \frac{1}{2}\right) \tilde{x}_{3}}{\left(3,3, \frac{2}{3}, \frac{2}{3}\right) \tilde{x}_{1}+(2,3,0,0) \tilde{x}_{2}+\left(1,2, \frac{1}{2}, \frac{1}{2}\right) \tilde{x}_{3}}$

Subject to

$$
\left(0,2, \frac{1}{2}, \frac{1}{2}\right) \tilde{x}_{1}+(1,3,1,1) \tilde{x}_{2}+\left(0,2, \frac{1}{2}, \frac{1}{2}\right) \quad \tilde{x}_{3}
$$$$
(1,3,1,1) \tilde{x}_{1}+\left(0,2, \frac{1}{2}, \frac{1}{2}\right) \bar{x}_{2}+(2,4,1,1) \tilde{x}_{3}
$$

$\preccurlyeq(3,5,1,1)$ 
Converting to

$$
\tilde{x}_{1}, \tilde{x}_{2}, \tilde{x}_{3} \geqslant \tilde{0}
$$

$\operatorname{Max} \tilde{z}_{1}=\left(4,6, \frac{1}{3}, \frac{1}{3}\right) \tilde{x}_{1}+(5,6,1,1) \tilde{x}_{2}$

Subject to

$$
+\left(5,6, \frac{1}{2}, \frac{1}{2}\right) \tilde{x}_{3}
$$

$\preccurlyeq(2,4,1,1)$

$$
\left(0,2, \frac{1}{2}, \frac{1}{2}\right) \tilde{x}_{1}+(1,3,1,1) \tilde{x}_{2}+\left(0,2, \frac{1}{2}, \frac{1}{2} \tilde{x}_{3}\right.
$$

$\preccurlyeq(3,5,1,1)$

$$
(1,3,1,1) \tilde{x}_{1}+\left(0,2, \frac{1}{2}, \frac{1}{2}\right) \tilde{x}_{2}+(2,4,1,1) \tilde{x}_{3}
$$

$$
\tilde{x}_{1}, \tilde{x}_{2}, \bar{x}_{3} \geqslant \tilde{0}
$$

And

$\operatorname{Min} \tilde{z}_{2}=\left(3,3, \frac{2}{3}, \frac{2}{3}\right) \tilde{x}_{1}+(2,3,0,0) \tilde{x}_{2}$

Subject to

$$
+\left(1,2, \frac{1}{2}, \frac{1}{2}\right) \tilde{x}_{3}
$$

$\preccurlyeq(2,4,1,1)$

$$
\left(0,2, \frac{1}{2}, \frac{1}{2}\right) \tilde{x}_{1}+(1,3,1,1) \tilde{x}_{2}+\left(0,2, \frac{1}{2}, \frac{1}{2}\right) \tilde{x}_{3}
$$

$$
(1,3,1,1) \tilde{x}_{1}+\left(0,2, \frac{1}{2}, \frac{1}{2}\right) \tilde{x}_{2}+(2,4,1,1) \tilde{x}_{3}
$$

$\preccurlyeq(3,5,1,1)$

$$
\tilde{x}_{1}, \tilde{x}_{2}, \tilde{x}_{3} \geqslant \tilde{0}
$$

Then

$\operatorname{Max} \tilde{z}_{*}=(1,3,1,1) \tilde{x}_{1}+(2,4,1,1) \tilde{x}_{2}+(3,5,1,1) \tilde{x}_{3}$

Subject to

$\preccurlyeq(2,4,1,1)$

$$
\left(0,2, \frac{1}{2}, \frac{1}{2}\right) \tilde{x}_{1}+(1,3,1,1) \tilde{x}_{2}+\left(0,2, \frac{1}{2}, \frac{1}{2}\right) \tilde{x}_{3}
$$

$$
(1,3,1,1) \tilde{x}_{1}+\left(0,2, \frac{1}{2}, \frac{1}{2}\right) \tilde{x}_{2}+(2,4,1,1) \tilde{x}_{3}
$$

$\preccurlyeq(3,5,1,1)$

See tables below.

$$
\tilde{x}_{1}, \tilde{x}_{2}, \tilde{x}_{3} \geqslant \tilde{0}
$$

$$
\begin{gathered}
\tilde{x}_{1}=0, \tilde{x}_{2}=\left(\frac{5}{2}, \frac{5}{2}, \frac{108}{35}, \frac{108}{35}\right), \tilde{x}_{3}=\left(\frac{-45}{8}, \frac{21}{8}, \frac{375}{56}, \frac{375}{56}\right) \\
\text { and } \tilde{z}_{*}=\left(\frac{-49}{12}, \frac{277}{12}, \frac{16267}{840}, \frac{16267}{840}\right)
\end{gathered}
$$

\section{Numerical example2:}

$\max \tilde{z}$

$$
\begin{gathered}
=\frac{(7,4,33) \tilde{x}_{1}+(10,9,6,6) \tilde{x}_{2}+(14,13,9,9)}{(1,5,2,2) \tilde{x}_{1}+(3,6,4,4) \tilde{x}_{2}+(5,9,6,6)} \\
\max \tilde{z}_{1}=(10,9,33) \tilde{x}_{1}+(10,13,3,3) \tilde{x}_{2} \\
\quad+(14,13,1,1) \\
\min \tilde{z}_{2}=(1,5,2,2) \tilde{x}_{1}+(3,4,4,4) \tilde{x}_{2}+(3,8,1,1) \\
\max \tilde{z}^{*}=(5,8,5,5) \tilde{x}_{1}+(6,10,7,7) \tilde{x}_{2} \\
\quad+(6,10,0,0)
\end{gathered}
$$

S.t $\quad(1,1,2,2) \tilde{x}_{1}-(1,2,3,3) \tilde{x}_{2} \preccurlyeq(1,-5,4,4)$

$$
\begin{gathered}
(-8,-2,1,1) \tilde{x}_{1}{ }^{\sim}+(2,2,1,1) \tilde{x}_{2} \preccurlyeq(7,3,3,3) \\
\tilde{x}_{1}, \tilde{x}_{2} \geq \tilde{0}
\end{gathered}
$$

See tables below.

$$
\begin{aligned}
& \tilde{x}_{1}=\left(3,3, \frac{1133}{28}, \frac{1133}{28}\right) \quad, \tilde{x}_{2}=\left(\frac{31}{4}, \frac{49}{4}, \frac{5217}{112}, \frac{5217}{112}\right) \\
& \text { and } \tilde{z}=\left(-63,142, \frac{19587}{28}, \frac{19587}{28}\right)
\end{aligned}
$$

\section{Conclusion}

The main aim of this paper is, is to solve (FFFLP) using the complementary development method to convert fuzzy fractional Linear programming problems to fuzzy Linear programming (FLP) problems and the method of dual simplex with the ranking of fuzzy number(FN) which is used partially in solving fuzzy linear programming.

\section{References}

[1]B.Stanojevic,M. Stanojevic (2013), "Solving Method for linear Fractional Optimization Problem with Fuzzy Coefficients in the Objective Function",INT $J$ COMPUT VOMMUX, S(1), 146-152.

[2] Tanaka, H., Okuda, T. and Asai, K. (1974), "On fuzzy mathematical programming", The Journal of Cybernetics, 13, $37-46$.

[3] Bellman, R.E. and Zadeh, L.A. (1970), "Decision making in a fuzzy environment", Management Science, 17, 141-164.

[4] T. Peric, Z . Babic and S. Resic, "A goal programming procedure for Solving fuzzy multiobjective fractional linear programming problems", Croatian Operational Research Review, vol.5, 2014, 401- 414.

[5] H. J. Zimmermann, "Fuzzy programming and linear programming with several objective functions", Fuzzy Sets and Systems 1 (1978) 45-55.

[6] S.C. Fang and C.F. Hu, "Linear programming with fuzzy coefficients in constraint", Compute. Math. Appl. 37 (1999) 63--76.

[7] P.Vasant, R.Nagarajan, and S. Yaacab, “Decision making in industrial production planning using fuzzy linear programming", IMA, Journal of Management Mathematics 15 (2004) 53--65.

[8] A.Nachammai and P.Thangaraj, Solving Intuitionist fuzzy linear Programming by using metric distance ranking, Department of Mathematics,Kongu Engineering College, vol.5, no.4, 2013, 65-70.

[9] H.M.Nehi and H.Hajmohamadi, "A ranking function method for solving fuzzy multi - objective linear programming problem ", a nnals of fuzzy Mathematics and Informatics, vol.3, no.1, 2012, 31-38.

[10] S.H .Nasseri and E.Ardil , Simplex method for fuzzy variable linear Programming problems, International Journal of Mathematical and Computational sciences, vol.1, no.10, 2007, 36-40. 


\begin{tabular}{|l|c|l|l|l|l|l|l|l|l|}
\hline ROW & basic & $\tilde{z}$ & \multicolumn{1}{|c|}{$\tilde{x}_{1}$} & $\tilde{x}_{2}$ & $\tilde{x}_{3}$ & $\tilde{x}_{4}$ & $\tilde{x}_{5}$ & RHS & R(R.H.S) \\
\hline 0 & $\tilde{z}$ & $\tilde{1}$ & $(-3,-1,1,1)$ & $(-4,-2,1,1)$ & $(-5,-3,1,1)$ & $\tilde{0}$ & $\tilde{0}$ & $\tilde{0}$ & \\
\hline 1 & $\tilde{x}_{4}$ & $\tilde{0}$ & $\left(-2,0, \frac{1}{2}, \frac{1}{2}\right)$ & $(-3,1,1,1)$ & $\left(-2,0, \frac{1}{2}, \frac{1}{2}\right)$ & $\tilde{1}$ & $\tilde{0}$ & $(-4,-2,1,1)$ & -6 \\
\hline 2 & $\tilde{x}_{5}$ & $\tilde{0}$ & $(-3,-1,1,1)$ & $\left(-2,0, \frac{1}{2}, \frac{1}{2}\right)$ & $(-4,-2,1,1)$ & $\tilde{0}$ & $\tilde{1}$ & $(-5,-3,1,1)$ & -8 \\
\hline
\end{tabular}

\begin{tabular}{|l|c|c|l|c|c|c|c|c|c|}
\hline $\begin{array}{l}\text { RO } \\
\text { W }\end{array}$ & basic & $\tilde{z}$ & $\tilde{x}_{1}$ & $\tilde{x}_{2}$ & $\tilde{x}_{3}$ & $\tilde{x}_{4}$ & $\tilde{x}_{5}$ & RHS & $\begin{array}{l}\text { R(R. } \\
\text { H.S })\end{array}$ \\
\hline 0 & $\tilde{z}$ & $\tilde{1}$ & $\left(\frac{-7}{2}, \frac{31}{6}, \frac{101}{24}, \frac{101}{24}\right)$ & $\left(\frac{-31}{6}, \frac{11}{6}, \frac{13}{12}, \frac{13}{12}\right)$ & $\tilde{0}$ & $\tilde{0}$ & $\left(\frac{-13}{6}, \frac{-1}{2}, \frac{77}{24}, \frac{77}{24}\right)$ & $\left(\frac{1}{2}, \frac{61}{6}, \frac{199}{24}, \frac{199}{24}\right)$ & \\
\hline 1 & $\tilde{x}_{4}$ & $\tilde{0}$ & $\left(\frac{-21}{8}, \frac{47}{24}, \frac{55}{48}, \frac{55}{48}\right)$ & $\left(\frac{-11}{3}, \frac{7}{3}, \frac{17}{12}, \frac{17}{12}\right)$ & $\tilde{0}$ & 1 & $\left(\frac{-19}{24}, \frac{1}{8}, \frac{5}{48}, \frac{5}{48}\right)$ & $\left(\frac{-39}{8}, \frac{37}{24} \frac{101}{48}, \frac{101}{48}\right)$ & $\frac{-80}{24}$ \\
\hline 2 & $x_{3}$ & $\tilde{0}$ & $\left(\frac{1}{24}, \frac{31}{24}, \frac{3}{2}, \frac{3}{2}\right)$ & $\left(\frac{-1}{6}, \frac{5}{6}, \frac{1}{4}, \frac{1}{4}\right)$ & $\tilde{1}$ & 0 & $\left(\frac{-11}{24}, \frac{-5}{24}, 1,1\right)$ & $\left(\frac{-85}{24}, \frac{53}{24}, \frac{7}{2}, \frac{7}{2}\right)$ & $\frac{-32}{24}$ \\
\hline
\end{tabular}

\begin{tabular}{|c|c|c|c|c|c|c|c|c|c|}
\hline ROW & $\begin{array}{l}\text { Basi } \\
\text { c }\end{array}$ & $\tilde{z}$ & $\tilde{x}_{1}$ & $\tilde{x}_{2}$ & $\tilde{x}_{3}$ & $\tilde{x}_{4}$ & $\tilde{x}_{5}$ & RHS & $\begin{array}{l}\mathrm{R}( \\
\mathrm{R} . \\
\mathrm{H} . \\
\mathrm{S})\end{array}$ \\
\hline 0 & $\overline{\tilde{Z}}$ & $\tilde{1}$ & $\left(\frac{-377}{48}, \frac{179}{16}, \frac{8507}{289}, \frac{8507}{289}\right)$ & $\tilde{0}$ & $\tilde{0}$ & $\left(\frac{-157}{18}, \frac{67}{18}, \frac{11}{216}, \frac{11}{216}\right)$ & $\left(\frac{-5245}{516}, \frac{4729}{516}, \frac{3277}{390}, \frac{3277}{390}\right)$ & $\left.\frac{-49}{12}, \frac{277}{12}, \frac{16267}{840}, \frac{16267}{840}\right)$ & \\
\hline 1 & $\tilde{x}_{2}$ & $\tilde{0}$ & $\left(\frac{-27}{56}, \frac{83}{56}, \frac{160}{49}, \frac{160}{49}\right)$ & $\tilde{1}$ & $\tilde{0}$ & $\left(\frac{-16}{9}, \frac{-11}{9}, \frac{17}{12}, \frac{17}{12}\right)$ & $\left(\frac{-52}{43}, \frac{95}{43}, \frac{2}{9}, \frac{2}{9}\right)$ & $\left(\frac{5}{2}, \frac{5}{2}, \frac{108}{35}, \frac{108}{35}\right)$ & + \\
\hline 2 & $x_{3}$ & $\tilde{0}$ & $\left(\frac{-317}{336}, \frac{653}{336}, \frac{877}{191}, \frac{877}{191}\right)$ & $\tilde{0}$ & $\tilde{1}$ & $\left(\frac{7}{18}, \frac{25}{18}, \frac{7}{8}, \frac{7}{8}\right)$ & $\left(\frac{-705}{344}, \frac{361}{344}, \frac{139}{80}, \frac{139}{80}\right)$ & $\left(\frac{-45}{8}, \frac{21}{8}, \frac{375}{56}, \frac{375}{56}\right)$ & + \\
\hline
\end{tabular}

\begin{tabular}{|c|c|c|c|c|c|c|c|c|}
\hline ROW & BASIC & $\tilde{Z}$ & $\tilde{x}_{1}$ & $\tilde{x}_{2}$ & $\tilde{x}_{3}$ & $\tilde{x}_{4}$ & RHS & \\
\hline 0 & $\tilde{Z}$ & $\tilde{1}$ & $(-8,-5,5,5)$ & $\begin{array}{c}(-10,- \\
6,10,10)\end{array}$ & $\tilde{0}$ & $\tilde{0}$ & $\tilde{0}$ & 0 \\
\hline 1 & $\widetilde{x}_{3}$ & $\tilde{0}$ & $(1,1,2,2)$ & $(-2,1,3,3)$ & $(1,1,0,0)$ & $\tilde{0}$ & $(1,-5,4,4)$ & -4 \\
\hline 2 & $\tilde{x}_{4}$ & $\tilde{0}$ & $(-8,-2,1,1)$ & $(2,2,1,1)$ & $\tilde{0}$ & $\begin{array}{c}(1,1,0,0 \\
)\end{array}$ & $(7,3,3,3)$ & 10 \\
\hline & $\mathrm{R}\left(z_{j}{ }^{\sim}\right)$ & & & 16 & $\tilde{0}$ & $\tilde{0}$ & $\tilde{0}$ & \\
\hline
\end{tabular}

\begin{tabular}{|c|c|c|c|c|c|c|c|c|}
\hline ROW & BASIC & $\tilde{Z}$ & $\tilde{x}_{1}$ & $\tilde{x}_{2}$ & $\tilde{x}_{3}$ & $\tilde{x}_{4}$ & RHS & A \\
\hline 0 & $\tilde{Z}$ & $\tilde{1}$ & $\left(-34,13, \frac{45}{2}, \frac{45}{2}\right.$ & $\tilde{0}$ & $\begin{array}{c}\left(-26,18, \frac{11}{2}\right. \\
\left., \frac{11}{2}\right)\end{array}$ & $\tilde{0}$ & $\begin{array}{c}(- \\
24,40,106,10 \\
6)\end{array}$ & \\
\hline 1 & $\tilde{x}_{2}$ & $\tilde{0}$ & $\left(\frac{-11}{4}, \frac{-5}{4}, 5,5\right)$ & $\tilde{1}$ & $\begin{array}{c}\left(\frac{-11}{4}, \frac{-5}{4}, 3,\right. \\
3)\end{array}$ & $\tilde{0}$ & $(4,4,11,11)$ & 8 \\
\hline 2 & $\tilde{x}_{4}$ & $\tilde{0}$ & $\left(\frac{-11}{2}, \frac{7}{2}, \frac{39}{4}, \frac{39}{4}\right)$ & $\tilde{0}$ & $\begin{array}{c}\left(\frac{5}{2}, \frac{11}{2}, \frac{19}{4},\right. \\
\left.\frac{19}{4},\right)\end{array}$ & $\tilde{1}$ & $(-1,-5,29,29)$ & -6 \\
\hline & $\mathrm{R}\left(z_{j}{ }^{\sim}\right)$ & & & & & & & \\
\hline
\end{tabular}

\begin{tabular}{|c|c|c|c|c|c|c|c|c|}
\hline ROW & BASIC & $\tilde{Z}$ & $\tilde{x}_{1}$ & $\tilde{x}_{2}$ & $\tilde{x}_{3}$ & $\tilde{x}_{4}$ & RHS & \\
\hline 0 & $\tilde{Z}$ & $\tilde{1}$ & $\tilde{0}$ & $\tilde{0}$ & $\left(\frac{-2691}{14},-\frac{1403}{14}, \frac{29969}{28}, \frac{29969}{28}\right)$ & $\left(-33,14, \frac{417}{4}, \frac{417}{4}\right)$ & $\left(-63,142, \frac{19587}{28}, \frac{19587}{28}\right)$ & \\
\hline 1 & $\tilde{x}_{2}$ & $\tilde{0}$ & $\tilde{0}$ & $\tilde{1}$ & $\left(\frac{-457}{28}, \frac{-103}{28}, \frac{13981}{112}, \frac{13981}{112}\right)$ & $\left(\frac{-7}{4}, \frac{-1}{4}, \frac{275}{16}, \frac{275}{16}\right)$ & $\left(\frac{31}{4}, \frac{49}{4}, \frac{5217}{112}, \frac{5217}{112}\right)$ & \\
\hline 2 & $\tilde{x}_{1}$ & $\tilde{0}$ & $\tilde{1}$ & 0 & $\left(\frac{-37}{7}, \frac{-19}{7}, \frac{2425}{28}, \frac{2425}{28}\right)$ & $\left(-1,-1, \frac{39}{4}, \frac{39}{4}\right)$ & $\left(3,3, \frac{1133}{28}, \frac{1133}{28}\right)$ & \\
\hline & $\mathrm{R}\left(z_{j} \sim\right)$ & & & & & & \\
\hline
\end{tabular}


Journal of the University of Garmian 6 (1), 2019 